\title{
In Science We Trust: Überlegungen zum Wissen der Wissenschaften
}

\begin{abstract}
It is far more judicious to trust in scientificity than in science, namely that research follows the rules of rationality, and that analysis and interpretation are constantly monitored critically. The sciences are not defined by their truth claims, but by their constant self-criticism as the driving force behind all progress to produce more and better knowledge. In this respect, there is hardly any difference between the natural sciences and the humanities.

Zusammenfassung: Vertrauen gebührt weniger den Wissenschaften als der Wissenschaftlichkeit: dass Forschung nach rationalen Regeln verfährt und Analyse wie Interpretation der Kontrolle unterliegen. Nicht der Anspruch auf Wahrheit macht daher Wissenschaft aus, sondern die beständige Selbstkritik als entscheidender Motor allen Fortschritts $\mathrm{zu}$ immer besserer Erkenntnis. In dieser Hinsicht unterscheiden sich Natur- und Geisteswissenschaften nur unwesentlich voneinander.
\end{abstract}

\begin{abstract}
Wenn Gott in seiner Rechten alle Wahrheit, und in seiner Linken den einzigen immer regen Trieb nach Wahrheit, obschon mit dem Zusatze, mich immer und ewig zu irren, verschlossen hielte, und spräche zu mir: wähle! Ich fiele ihm mit Demuth in seine Linke, und sagte: Vater gieb! die reine Wahrheit ist ja doch nur für dich allein!
\end{abstract}

Gotthold Ephraim Lessing (1778)

In Science we trust - namentlich in den USA ist das zu einem allseits beliebten Schlagwort geworden, das sich auf Demonstrationen nicht weniger gut macht als auf Designer T-Shirts. Was daran zum Ausdruck kommen soll, steht außer Frage, und kaum jemand wird sich zurückhalten wollen, wenn es um den Protest gegen jeglichen Irrationalismus geht, der als fake news, als alternative facts oder gar als junk science auftritt.

Einem Literaturwissenschaftler wird bei der Parole In Science we trust dennoch nicht wohl, weil die Unfähigkeit, einen Satz schlechthin beim Wort zu nehmen, zu den Berufskrankheiten gehört: Jede Aussage lässt Vorlagen respektive Muster durchscheinen und relativiert sich dadurch - als Zitat - in objektiver Ironie selbst. Bei In Science we trust drängt sich nun das Original, das einen jeden Dollar-Schein ziert, geradezu überdeutlich auf: In God we trust. Kann bzw. darf man auf die Wissenschaft heute aber in der gleichen Weise vertrauen wie früher einmal auf den Schöpfer aller Dinge? Ist die Wissenschaft tatsächlich die oberste Sinn-Instanz unserer Zeit, an deren Allmacht man glauben müsste: bei Strafe zwar nicht ewiger Höllenpein, doch gründlicher, weil freiwilliger Verdummung? 


\section{Wahrheit}

Nicht-Fachwissenschaftler sind kaum je in der Lage, mit eigenen Mitteln zu entscheiden, ob ein bestimmtes Forschungsergebnis wirklich schlüssig ist und als gültige Erkenntnis Bestand haben wird. Wer von einer Sache nicht genug versteht, hat folglich nolens volens auf diejenigen zu hören, die von Amts wegen über die erforderliche Expertise verfügen, denn der ,gesunde Menschenverstand' reicht in Dingen der Wissenschaft schon lange nicht mehr weit. Laien bleibt insofern keine Wahl: Sie müssen sich auf die Spezialisten verlassen und sind dabei auf deren intellektuelle wie moralische Redlichkeit angewiesen. Demgemäß stellt sich das Verhältnis zwischen dem Sozialsystem Wissenschaft und der fachwissenschaftlich laienhaften Gesellschaft in der Tat als eine religionsähnliche Beziehung dar. In Science we trust bringt diese buchstäblich ,katholische“ Konstellation auf den Punkt und weist zugleich auf das hin, was daran bedenklich ist: Wissenschaft schafft Wissen, und das, was man weiß, ist per definitionem ,wahr`. Die Rede vom ,Wissen' unterstellt mithin die Zuverlässigkeit der jeweiligen Aussage und setzt dabei voraus, dass die entsprechende Überzeugung von Fall zu Fall begründet werden kann. Ist etwas nun tatsächlich wahr, dann wird sich auch von anderen verlangen lassen, dass sie diese Wahrheit als solche hinnehmen, das Wissen in seiner Objektivität anerkennen und sich ihm anschließen. Wer das Wissen hat, der muss folglich im Recht sein (und zwar allein auf seine Weise). Die menschliche Vernunft ist in solchen Dingen unerbittlich.

Überall dort, wo es auf solche Glaubens- bzw. Wissensfrömmigkeit ankommt, liegt allerdings auch der Zweifel nicht fern, und spätestens seit dem 18. Jahrhundert sind gute Gründe genug bekannt, um den Autoritäten mit ihren heiligen Büchern nicht ungeprüft alles nachzureden. In Dingen der Wissenschaft gilt das erst recht angesichts der leidvollen Erfahrung, dass die Fach-Kapazitäten meist ebenso wenig einer einzigen Meinung sind wie sonst nur die Theologen (die beständigen Nachweise, dass Kaffee oder Salz der Gesundheit schaden bzw. doch nicht, zeigen das in gewohnter Regelmäßigkeit). Über kurz oder lang wird jeder Glaube also nach Widerspruch verlangen. In diesem Interesse muss es folglich Ketzer geben, und schon gar nicht wird es ohne falsche Propheten abgehen können.

\section{Vernunft}

Wenn alle Aufklärung, wie es bei Immanuel Kant (1784: 481) heißt, im „Ausgang des Menschen aus seiner selbst verschuldeten Unmündigkeit“ besteht, dann kann der ureigenste Anspruch menschlicher Vernunft nur auf eines hinauslaufen: sapere aude! statt credere! oder gar confidere! Das entscheidende Wort dabei ist nicht sapere, sondern aude!, da jedes Wissen mit dem Wagnis des Irrtums verbunden ist und folglich ganz entschieden nach dem Mut zur beständigen Skepsis verlangt, speziell hinsichtlich der eigenen Meinungen und ihrer Bedingtheit. Hier wurzeln nun zuallererst 
die Probleme im Verhältnis dessen, was man gewöhnlich noch immer kontrastierend ,die Naturwissenschaften' und ,die Geisteswissenschaften“ nennt. Salopp lässt sich das folgendermaßen auf den Punkt bringen: Naturwissenschaftler müssen es in jedem Fall richtigmachen und zielen daher auf positives, das heißt dauerhaft gültiges Wissen ab; sie werden dabei jedoch umso leichter und häufiger falsifiziert, weil ihre Erkenntnisse in aller Regel nicht schon richtig genug sind. In den Geisteswissenschaften genügt es demgegenüber oft schon, wenn man einigermaßen plausibel zu sagen weiß, woran eine Theorie krankt oder warum man ein bestimmtes Problem vielleicht überhaupt nicht lösen kann. Die Geisteswissenschaften haben es folglich gut, weil sie nicht leicht zu widerlegen sind, sondern höchstens überholt; mit ihrer praktischen Belanglosigkeit zahlen sie für diese Bequemlichkeit allerdings teuer, denn sehr viel leichter ließe sich auf Byzantinistik verzichten denn auf Krebsforschung.

\section{Realismus}

Insbesondere im späten 20. Jahrhundert ist Kants berühmte Frage nach dem, was wir eigentlich ,wissen können ', ${ }^{1}$ gern in kontrapunktischer Weise beantwortet worden:

a) von naturwissenschaftlicher Warte aus unter der optimistischen Prämisse eines gewissermaßen objektiven Realismus, demzufolge wir die Welt mit all ihren Eigenheiten letztlich einigermaßen richtig durchschauen werden; zwar nie ganz und gar, nach dem Muster einer Intervall-Schachtelung aber immer besser;

b) von geisteswissenschaftlicher Warte aus mit dem pessimistisch-skeptischen Schlagwort von der ,Konstruiertheit‘ aller Wissensinhalte und -formen, demgemäß all unser Wissen unmöglich ,an sich' gelten kann, sondern stets auf die eine oder andere Weise durch gesellschaftliche und/oder anthropologische Rahmenumstände geprägt ist.

Mit anderen Worten: Wenn man Geistes- und Naturwissenschaften mit einander vergleicht, dann mag es auf den ersten Blick so aussehen, als würden sich ein prinzipieller Relativismus und ein ebenso prinzipieller Absolutismus gegenüberstehen, indem es entweder mehrere, nicht gegeneinander ausspielbare Wahrheiten von je begrenzter Reichweite geben soll oder immer nur die einzige Wahrheit, die dann für jeden vernünftigen Menschen zwingend sein müsste.

1 „Alles Interesse meiner Vernunft (das speculative so wol, als das practische) vereinigt sich in folgenden drey Fragen: | 1. Was kan ich wissen? | 2. Was soll ich thun? | 3. Was darf ich hoffen?“ (Kant 1781: 804-805). 


\section{Relativismus}

Innerhalb der Geisteswissenschaften hat es lange als Grundregel gegolten, Wissen um keinen Preis zu verabsolutieren und auf jeden Fall zu verhindern, dass eine bestimmte Wissensform (immer die okzidentale Rationalität) sich über andere, konkurrierende erhebt. Der US-amerikanische Philosoph Paul Feyerabend hat in diesem Sinn provokativ behauptet, es gebe sehr wohl unterschiedliche Arten von Wissenschaft und Wissenschaftlichkeit, und gewiss nicht $\mathrm{zu}$ Unrecht betont, dass eine Privilegierung abendländischer Konzepte alles andere als unschuldig wäre, indem sie gar zu schnell in ein Instrument politischer Unterdrückung umschlüge: „First-world science is one science among many; by claiming to be more it ceases to be an instrument of research and turns into a (political) pressure group“ (Feyerabend 2010 [1975]: XXI). Vor diesem Hintergrund hat ein am 22. Oktober 1996 in der New York Times erschienener Artikel (Johnson 1996) vorübergehend für Irritationen gesorgt: Archäologen können offenbar mit evidenzbasierten Gründen belegen, dass die nordamerikanischen ,Ureinwohner gar nicht autochthon sind, sondern um das Jahr 8000 v. Chr. über die Bering-Straße eingewandert sein müssen; im Großen und Ganzen scheint es gegen diese Theorie keine wissenschaftlichen Bedenken $\mathrm{zu}$ geben. $\mathrm{Zu}$ den Ursprungsmythen etwa der Cheyenne River Sioux gehört demgegenüber der Glaube, dass ihre Urväter einst an Ort und Stelle aus einer unterirdischen Geisterwelt hervorgegangen seien, ${ }^{2}$ und der britische Archäologe Roger Anyon hat diese Sichtweise als „ebenso valide“ bezeichnet wie die „archäologische Sichtweise auf das, was die Vorgeschichte ausmacht“. ${ }^{3}$ Sein Kollege Larry Zimmerman geht in gleicher Weise von der Notwendigkeit einer „anderen Art von Wissenschaft zwischen den Grenzen der westlichen Art des Erkennens und den indianischen Arten des Erkennens" aus; er wehrt sich aus diesem Grund gegen das Ansinnen, unsere rationale „Wissenschaft als eine höherrangige Weise des Blicks auf die Welt“ zu privilegieren. ${ }^{4}$ Solchen Überlegungen scheint nun genau jener Relativismus zugrunde zu liegen, der in den Geisteswissenschaften lange Zeit als regulative Idee aller Wissenschaftsethik gegolten hat und nach wie vor im heiklen

2 „We never asked science to make a determination as to our origins. [...] We know where we came from. We are the descendants of the Buffalo people. They came from inside the earth after supernatural spirits prepared this world for humankind to live here. If non-Indians choose to believe they evolved from an ape, so be it“" (zitiert nach Johnson 1996).

3 „,Science is one of many ways of knowing the world,' said Roger Anyon, a British archeologist who has worked for the Zuni tribe. The Zuni's world view, he said, is ,just as valid as the archeological viewpoint of what prehistory is about." (zitiert nach ebd.).

4 „Dr. Zimmerman of the University of Iowa said there was a need for ,a different kind of science, between the boundaries of Western ways of knowing and Indian ways of knowing.' I ,I personally do reject science as a privileged way of seeing the world', he said. ,That's not to say it isn't an important way that has brought benefit. But I understand that as a scientist I need to constantly learn. “ (zitiert nach ebd.). 
Gegensatz zu den Naturwissenschaften steht, die derartige Nonchalance wohl nur kopfschüttelnd zur Kenntnis nehmen können.

\section{An sich}

Seit einigen Jahren mehren sich in der Philosophie wie in den Literaturwissenschaften jedoch Stimmen, die wieder nach Eindeutigkeit und Entschiedenheit verlangen bzw. nicht mehr hinnehmen wollen, dass der einzige Zugang zu den Dingen über unsere Vorstellungen davon führen soll und wir folglich nie bis zum ,An-sich-Sein“ dieser Dinge gelangen könnten. ${ }^{5}$ Kernproblem ist dabei die Frage, ob es ,mind-independent facts ${ }^{6}$ im Sinne Hilary Putnams wirklich gibt, das heißt Dinge oder Sachverhalte, die ganz einfach so sind, wie sie sind, ohne dass menschliches Denken bzw. menschliches Wahrnehmen in irgendeiner belangvollen Weise dafür verantwortlich wäre. Weil Kants transzendentale Frage nach den Bedingungen der Möglichkeit des Denkens angesichts solcher Absolutheit des Seins gegenstandslos würde, glaubt der französische Realist Jocelyn Benoist „die Schließung dessen“ konstatieren zu dürfen, „was in der Philosophiegeschichte die kantsche Parenthese gewesen sein wird“.7

Für die Naturwissenschaften bzw. für diejenigen Wissenschaften, die man einst als die ,exakten' bezeichnet hat und heute eher ,harte' nennt, muss und darf der Glaube an mind-independent facts gewiss nach wie vor zur selbstverständlichen Voraussetzung dienen. In den Geisteswissenschaften aber kommt ungefähr seit dem Jahrtausendwechsel eine analoge Strömung immer deutlicher zur Geltung, die sich als ,Neuer Realismus ${ }^{\circ 8}$ ausgibt und all das zum Gegner erklärt, was sie allgemein als

5 „Sans doute un des lieux communs de l'épistémologie moderne a-t-il été et demeure-t-il que nous n'avons jamais accès aux choses qu'à travers leur représentation. [...] La métaphore de l'accès suppose qu'il y ait un chemin à parcourir pour aller jusqu'aux choses. Or, souvent, il est plutôt vrai, qu'elles viennent à nous.“ (Benoist 2011: 17 bzw. 2014: 17: „Ein Allgemeinplatz der modernen Erkenntnistheorie war und ist zweifelsohne, dass wir niemals anders Zugang zu den Dingen haben als durch deren Repräsentation. [...] Die Zugangsmetapher impliziert, dass es einen Weg gäbe, den man zurücklegen müsste, um bis zu den Dingen zu gelangen. Häufig jedoch trifft es eher zu, dass sie auf uns zukommen“).

6 "So what does ,mind-independent" mean? When I misguidedly used that expression in describing ,metaphysical realism', what I meant was that the metaphysical realist believes that there are truths that do not depend on whether human beings or other sentient beings could or could not verify them. Now, I do believe that there are such truths. Many cosmo-logical truths must be of that sort" (Putnam 2012: 100).

7 Benoist 2017: 16: „la clôture de ce qui aura été la parenthèse kantienne dans l'histoire de la philosophie“.

8 ,,...] il pendolo del pensiero, che nel Novecento inclinava verso l'antirealismo nelle sue varie versioni (ermeneutica, postmodernismo, ,svolta linguistica“ ecc.), con il tornante del secolo si era spostato verso il realismo“ (Ferraris 2012: IX bzw. 2014: 13: „Das Pendel des Denkens, das im 20. Jahrhundert Richtung Antirealismus und dessen verschiedene Formen (Hermeneutik, Postmodernismus, sprachliche Wende etc.) schwang, hatte sich um die Jahrhundertwende Richtung Realismus bewegt“). 
,Postmoderne‘ diskreditiert: ${ }^{9}$ summa summarum diejenige Denkweise, der man unterstellt, einen mehr oder weniger konsequenten Relativismus zu vertreten und - in der Nachfolge Friedrich Nietzsches ${ }^{\mathbf{1 0}}$ - zu leugnen, dass Wahrheit im eigentlichen Sinn uns Menschen überhaupt zugänglich ist.

Dem hält der Neue Realismus entgegen, dass es durchaus ,Wahrheit‘ gebe: als von menschlicher Interpretation unabhängige Tatsachen, an denen kein vernünftiges Denken vorbeikommt. In seinem Manifesto del nuovo realismo (2012) versucht der italienische Philosoph Maurizio Ferraris diese anti-relativistische Position an folgendem Beispiel zu erläutern: Wenn sich auf einem bestimmten Teppich ein bestimmter Pantoffel befindet, dann sei dies eine objektive, weil bewusstseinsunabhängige Tatsache. Auch nichtmenschliche Wesen wie etwa ein Hund, ein Wurm, ein Efeu-Zweig oder ein zweiter Pantoffel würden die physische, materielle Präsenz des Pantoffels daher anerkennen müssen, indem sie auf je ihre Weise darauf reagieren. So zwingend dieses Beispiel seinerseits ist, so wenig tut es freilich zur Sache, da die Frage nach der Menschenmöglichkeit von Wahrheit davon unbetroffen bleibt.

\section{Daten und Fakten}

Aufschlussreicher sollte es sein, zwischen Daten und Fakten terminologisch zu unterscheiden. ,Daten' sind das, was einem Beobachter buchstäblich gegeben ist (lat.: datum ( frz.: donnée) und daher insbesondere das, was sich in irgendeiner Weise feststellen und vielleicht sogar messen lässt. In erster Linie darf man also das als Datum verstehen, was niemand bestreiten kann, und mithin gilt ein Datum fraglos, sofern es korrekt ermittelt worden ist. Ein Faktum ist demgegenüber kein simples Datum, sondern wortwörtlich eine ,Tat-Sache‘, das heißt ein im Kern menschliches Produkt. Wo wir von Fakten reden, sprechen wir jedenfalls immer von Dingen, die wir selber erst zu dem gemacht haben, was sie sind (lat.: factum / frz.: fait). Können Daten insofern nicht anders als ,richtig' sein, solange man sie nicht irrtümlich oder sachwidrig erfasst hat, stellt sich bei Fakten von vornherein die Frage, inwiefern sie als ,wahr gelten dürfen.

\footnotetext{
9 „We have no choice but to recognize that there must be some objective, mind-independent facts“ (Boghossian 2006: 57).

10 „Was also ist Wahrheit? Ein bewegliches Heer von Metaphern, Metonymien, Anthropo-morphismen, kurz eine Summe von menschlichen Relationen, die, poetisch und rhetorisch gesteigert, übertragen, geschmückt wurden, und die nach langem Gebrauche einem Volke fest, canonisch und verbindlich dünken: die Wahrheiten sind Illusionen, von denen man vergessen hat, dass sie welche sind, Metaphern, die abgenutzt und sinnlich kraftlos geworden sind, Münzen, die ihr Bild verloren haben und nun als Metall, nicht mehr als Münzen in Betracht kommen“ (Nietzsche 1980 [1873]: 880-881).
} 
Um ein bloßes Datum handelt es sich etwa bei der Zusammensetzung von Wasser-Molekülen aus je zwei Wasserstoff-Atomen und einem Sauerstoff-Atom. ${ }^{11}$ Das ist überall so und nie anders gewesen, wird sich auf unendlich fernen Planeten in noch unbekannten Galaxien gleichermaßen verhalten und kann tel quel durch keinerlei kulturell-gesellschaftlich motivierte Beschreibung verändert werden. Ein ,Fakt‘ ist dieser - wissenschaftlich als $\mathrm{H}_{2} \mathrm{O}$ interpretierte - Sachverhalt dennoch nicht, wie sich an der Differenz zum Wasser deutlich genug ablesen lässt. In der Lebenswelt tritt Wasser nirgendwo schlechterdings als $\mathrm{H}_{2} \mathrm{O}$ auf, sondern ist durch organische wie anorganische Stoffe verunreinigt, stellt ein eventuell umkämpftes Lebensmittel dar und kann als Fluss Menschen trennen oder vereinen. In solchen Zusammenhängen ist Wasser also nicht einfach ,gegeben', sondern wird zu etwas gemacht oder richtiger gesagt: als etwas behandelt. Überall dort, wo es um wirkliches Wasser geht statt um die Formel in einem Schulbuch, kommt es insofern nicht als Datum, sondern als eine Tatsache vor, weil es ja erst durch menschliche Ausdeutung zu dem geworden worden ist, was es je an Ort und Stelle bedeutet.

Fakten sind vor diesem Hintergrund als bearbeitete bzw. ausgewertete Daten zu begreifen und können demzufolge a priori keine unproblematische Geltung beanspruchen. Für das Wissen der Wissenschaften heißt das zuallererst, dass es aus keiner Ansammlung von Daten besteht, sondern aus der Organisation von Tatsachen, die in aller Nüchternheit als ,konfigurierte' Daten zu behandeln sind, deren Wahrheitswert stets fragwürdig bleibt. Dass man sich bei allen Fakten den leidigen Rattenschwanz von Subjektivität, Partikularinteressen, ideologischen Vorbehalten etc. einhandelt, versteht sich mithin von selbst, und festzuhalten gilt es umso mehr, dass Tatsachen als solche keineswegs über jeden Zweifel erhaben sind. Jeder Tatsache ist vielmehr schon ihre Bewertung eingeschrieben, über die sich dann gegebenenfalls streiten lässt. Insoweit gilt das für jegliches Faktum und folglich auch für jedes Wissen unserer Wissenschaften, das sich in Tatsachenbehauptungen konkretisiert.

Die verschiedenen Fachkulturen werden mit dieser Gegebenheit auf unterschiedliche Weise umzugehen haben. Die Natur- und Technikwissenschaften arbeiten im Regelfall zentripetal, weil es für sie idealiter auf die eine, einzig richtige Erkenntnis bzw. Lösung ankommt (und realiter zumindest auf die jeweils ,beste`); Geisteswissenschaften können sich demgegenüber nicht selten den Luxus erlauben, eher zentrifugal zu arbeiten, und das heißt konkret ,negativ‘, indem sie eben nicht versuchen, etwas so Heikles wie den Sinn eines gegenstandslosen Gemäldes allgemeingültig festzulegen, sondern lieber danach fragen, aus welchen Gründen sich das verbietet.

11 Dass diese Atome nach heutigem Wissen aus allen möglichen Elementarteilchen bestehen und diese wiederum aus noch elementareren Teilchen, darf hier außer Acht bleiben. 


\section{Anzestralität}

Diese Differenz lässt sich an einem Themenbereich illustrieren, der den französischen Philosophen Quentin Meillassoux zu seinem ,spekulativen Realismus' motiviert hat, mit dessen Hilfe er das geisteswissenschaftliche Axiom des sogenannten Korrelationismus ${ }^{12}$ aushebeln will: die traditionelle Annahme, dass uns reine Objektivität ganz grundsätzlich verschlossen sei, weil wir alle Dinge ja in unserem Bewusstsein erfassen; folglich hätten wir es bei jeder Weltwahrnehmung ausnahmslos mit unseren eigenen Gedanken zu tun, nicht mit den Dingen als solchen. ${ }^{13}$ Das Problem ist nun aber, dass hochtechnisierte Wissenschaften wie die Astrophysik oder die Paläontologie mittlerweile in der Lage sind, auch über solche Sachverhalte und Vorgänge gut begründete Aussagen zu machen, die weit vor die Zeit zurückreichen, seit der es irgendein bewusstes, vielleicht gar vernünftiges Leben und damit Subjektivität und Wahrnehmung überhaupt gibt. ${ }^{14}$ Meillassoux nennt diese Realität, die offenbar schon undenklich lange vor dem Auftreten des Menschen wirklich war, , anzestral' ${ }^{15}$

Naturwissenschaftler werden mit solcher Anzestralität keinerlei Problem haben, Geisteswissenschaftler hingegen schon, weil ihnen eine vollkommen geistlose Wirklichkeit schlechterdings nicht denkbar ist. Denn in der Tat: Wer zum Beispiel vom Urknall redet, der begibt sich automatisch in die Position eines Beobachters, der damals dabei gewesen ist und nun als vertrauenswürdiger Zeuge davon erzählt. ${ }^{16}$ Die logische Zumutung hier ist mit Händen zu greifen, das philosophische Problem aber nicht von der Hand zu weisen, wenngleich die Naturwissenschaft es getrost ignorieren mag.

12 „Par ,corrélation', nous entendons l'idée suivant laquelle nous n’avons accès qu'à la corrélation de la pensée et l'être, et jamais à l'un de ces termes pris isolément. Nous ap-pellerons donc désormais corrélationisme tout courant de pensée qui soutiendra le caractère indépassable de la corrélation ainsi entendue“ (Meillassoux 2006: 18 bzw. 2008: 18: „Unter ,Korrelation“ verstehen wir die Idee, derzufolge wir Zugang nur zu einer Korrelation von Denken und Sein haben, und nie gesondert zu einem der beiden Begriffe. Daher nennen wir von jetzt an Korrelationismus jede Denkrichtung, welche den unüberschreitbaren Charakter der so verstandenen Korrelation vertritt“).

13 „Le corrélationisme consiste à disqualifier toute prétention à considérer les sphères de la subjectivité et de l'objectivité indépendamment l'une de l'autre“ (ebd.: 19 bzw. 18: „Der Korrelationismus besteht in der Zurückweisung aller Versuche, die Sphären der Subjektivität und der Objektivität unabhängig voneinander zu denken“).

14 „La science expérimentale est aujourd'hui capable de produire des énoncés concernant des événements antérieurs à l'avènement de la vie comme de la conscience“ (ebd.: 24 bzw. 23: „Die experimentelle Wissenschaft ist heute in der Lage, Aussagen zu treffen, die sich auf Ereignisse beziehen, die vor der Entstehung des Lebens sowie der Entstehung des Bewusstseins liegen“).

15, „[...] nous nommons ancestrale toute réalité antérieure à l'apparition de l'espèce humaine - et même antérieure à toute forme recensée de vie sur la Terre“ (ebd.: 26 bzw. 24: „Wir nennen anzestral jede Wirklichkeit, die dem Aufkommen der menschlichen Gattung vorausgeht - und die sogar jeder erfassten Form des Lebens auf der Erde vorausgeht“).

16 „Wenn immer man denkt oder sagt: es ,gibt‘ eine Sache, es ,gibt“ eine Welt, und damit mehr meint als nur, es gibt etwas, das ist, wie es ist, dann ist ein Beobachter involviert“ (Luhmann 1990: 62). 
Nun ist Meillassoux aber ein Philosoph und findet als solcher seinen philosophischen Ausweg: Es ist gar nicht erforderlich, dem Problem eine Lösung zu geben, da es - zumindest vorläufig - schon genügt, es aufgeworfen zu haben. ${ }^{17}$ Am Problem der Anzestralität wird in der Tat schlagend klar, dass der seit Kant etablierte Korrelationismus in seiner klassischen Ausprägung nicht länger haltbar ist und wir in manchen Fällen etwas ,Absolutes‘, vom Menschen in jeder Hinsicht Unabhängiges denken müssen. ${ }^{18}$

\section{Metaphysik}

Überschätzen sollte man die Unterschiede zwischen Natur- und Geisteswissenschaften trotzdem nicht. Auch bei Letzteren handelt es sich zu einem großen Teil um Erfahrungswissenschaften, weil sie konkrete Sachverhalte wie ein historisches Ereignis, einen sozialen Widerspruch oder zum Beispiel auch die durchaus nicht zufällige Entwicklungsgeschichte der literarischen Gattung ,Roman' erforschen. In all dem lassen sich ebenso Gesetzmäßigkeiten aufzeigen wie etwa beim Wetter, ohne dass diese Einsicht freilich dazu verleiten dürfte, literarische Ereignisse der Zukunft vorhersagen zu wollen.

Umgekehrt sind auch die Naturwissenschaften per se metaphysisch fundiert, ${ }^{19}$ und zwar aus einem zumindest doppelten Grund: zum einen, weil sie notwendig in weltanschaulichen Zusammenhängen stehen und daher im Rahmen von Paradigmen funktionieren, weshalb es nicht gleichgültig ist, zu welcher Weltanschauung sich ein Physiker bekennt oder ob er ein Fernrohr bzw. einen Teilchenbeschleuniger für hilfreich hält; zum anderen aufgrund der Notwendigkeit, diese Abhängigkeit von außer-

17 „Nous commençons à saisir que l'ancestralité constitue un problème philosophique, susceptible de nous faire réviser des décisions souvent considérées comme infrangibles depuis Kant. Mais disons-le tout de suite: notre ambition n'est pas ici de résoudre un tel problème, seulement de tenter de le poser sous une forme rigoureuse, et cela de telle sorte que sa résolution cesse de nous apparaitre tout à fait impensable“ (Meillassoux 2006: 48 bzw. 2008: 44: „Wir fangen an zu begreifen, dass die Anzestralität ein philosophisches Problem bildet, das uns in die Lage versetzt, Entscheidungen, die seit Kant oft als unhintergehbar betrachtet werden, zu revidieren. Doch sagen wir es gleich: Unser Anspruch ist hier nicht, solch ein Problem zu lösen, wir versuchen nur, es in einer strengen Form zu stellen, und das auf eine solche Weise, dass uns seine Lösung nicht länger ganz undenkbar erscheint“). 18 „Si le problème de Hume a réveillé Kant de son sommeil dogmatique, il reste à espérer que le problème de l'ancestralité nous réveille de notre sommeil corrélationnel, en nous engageant à réconcilier pensée et absolu“ (ebd.: 190 bzw. 171-172: „Wenn Kant durch das Problem Humes aus seinem dogmatischen Schlummer geweckt wurde, dann bleibt zu hoffen, dass das Problem der Anzestralität uns aus unserem korrelationellen Schlummer weckt und wir danach streben, das Denken mit dem Absoluten wieder zu versöhnen“).

19 „Ein metaphysischer Rest liegt darin, daß die Naturwissenschaftler die Natur für konkret halten und die Abstraktion übersehen, in der ihre Natur zum wissenschaftlichen Thema gestaltet worden ist“ (Husserl 2012 [1954]: 247). 
wissenschaftlichen Faktoren ${ }^{20}$ selbst mit ins Kalkül zu ziehen, das heißt sich eben diejenigen Horizonte bewusst $\mathrm{zu}$ machen, vor denen Wissenschaftler arbeiten und vor denen ihre Erkenntnisse fruchtbar werden.

Wie die Naturwissenschaften leisten auch die Geisteswissenschaften neben ihrer unmittelbar gegenstandsbezogenen, zweckhaften Arbeit zugleich Grundlagenforschung, wo es letztlich um ihre eigenen Voraussetzungen geht: Was auf der einen Seite vielleicht die theoretische Physik oder die Mathematik sein mag, das ist auf der anderen etwa die Semiotik, in der Jacques Derridas Dekonstruktion für unser Bewusstsein von Sprache Ähnliches bewirkt hat wie Werner Heisenbergs UnschärfeRelation für unser Verständnis von der materiellen Welt.

\section{Widerstreit}

Wissenschaftliches ,Wissen“ ist insofern nicht unbedingt das, was sich in ,wahren“ Erkenntnissen konkretisiert. Seine besondere Leistungsfähigkeit erklärt sich vielmehr daraus, dass dieses Wissen im Rahmen methodisch reflektierter Verfahren entwickelt und in seinen Resultaten geprüft wird, sodass jeweils gute Gründe anzuführen sind, warum die eine Einsicht Geltung erlangt und die anderen nicht. Von dieser Prämisse ausgehend, lässt sich auch das angesprochene Abstammungsproblem nordamerikanischer Indianer einer Lösung zuführen, in der sich naturwissenschaftliche und geisteswissenschaftliche Strategien vereinen:

Solange man die wissenschaftliche Theorie von der einstigen Einwanderung über die Bering-See nicht mit Argumenten vom gleichen Schlag widerlegen kann, solange steht es völlig außer Frage, dass sie als historische - und das heißt: als tatsächliche - Erkenntnis gelten darf. Derzeit lassen die archäologischen Zeugnisse daran ebenso wenig Widerspruch zu wie die genetischen Befunde. An solcher rationalen Faktizität gemessen, ist die mythische Erzählung von der autochthonen Entstehung des Buffalo-Volks ganz einfach abwegig und muss - guten Willen vorausgesetzt - auch von den Betroffenen selbst als unwahr eingesehen werden können. Zugleich aber lässt sich gerade von rationaler Warte aus begreifen, wie unverzichtbar es für das Selbstverständnis einer lokalen Kultur sein mag, die eigene Tradition nicht anzutasten, weil nur sie Identität im Sinne von Selbstgewissheit garantiert. Das ist dann zwar kein gleichartiges Wissen und schon gar keine gleichrangige Wissenschaft, sondern etwas Anderes: Glaube anstelle von Wissen bzw. Dichtung anstelle von Wissenschaft. So unterschiedlich beide Methoden auch sind, können sie allerdings die gleiche Leistung erbringen: nämlich das Dasein der nordamerikanischen Ureinwohner überzeugend erklären. Es ist daher durchaus vernünftig und steht nicht im Widerspruch zu den Ansprüchen westlicher Vernunft, wenn man die indianische Auffassung in ihrem

20, „[...] factual judgments, even in physics, depend on and presuppose epistemic values“ (Putnam 2012: 291). 
spezifischen Recht bzw. in ihrer Eigengesetzlichkeit respektiert. ${ }^{21}$ Dass manche englischsprachigen Archäologen bereit sind, Wissenschaft nur als ,one of many ways of knowing the world“ zu relativieren, klingt in deutschen Ohren ohnehin skandalöser als nötig: to know heißt nicht nur wissen, sondern zugleich auch erkennen. Eigentliches Wissen über die tatsächlichen Vorgänge vor gut 10.000 Jahren kann allein die Wissenschaft mit ihren überprüfbaren Methoden liefern und nicht der indianische Mythos; eine schlüssige Erklärung der Welt liefern jedoch beide in Übereinstimmung mit den je spezifischen Ansprüchen, die an solche Erklärungen gestellt werden.

Wissenschaft und Mythos lassen sich insofern nicht derart gegeneinander verrechnen, dass die eine Erklärungsweise unbedingt besser wäre als die andere und diese daher notwendig ausschließen müsste. Beide Denkweisen stehen vielmehr in einem klassischen ,Widerstreit', ${ }^{22}$ weshalb ihre Gegensätzlichkeit kein logisches Problem zur Folge hat, sondern mit abendländischer Wissenschaft nicht zuletzt aus dem Grund harmoniert, dass sie rational zu beschreiben ist (allerdings wohl mehr in Gestalt geisteswissenschaftlicher Analysen als in der eines naturwissenschaftlichen Fazits). Wissen und Glauben müssen sich dann nicht unbedingt ausschließen, wenn sie in ihren konkreten Grenzen bzw. Voraussetzungen zur Geltung kommen, wozu auch immer gehört, die jeweilige Funktion ins Kalkül einzubeziehen.

\section{Kontextualismus}

In dieser Hinsicht dürfte es hilfreich sein, auf das Konzept des ,Kontextualismus“ zurückzugreifen, mit dem Jocelyn Benoist versucht, über den postmodernen Korrelationismus hinauszugelangen. Ihm zufolge gibt es das Wirkliche grundsätzlich immer nur in Zusammenhängen einer ,Intentionalität' und daher komme es grundsätzlich auf das an, was unter der jeweiligen Bedingung zählt und was nicht. Nehmen wir das Beispiel eines Tisches: Im Kontext menschlicher Alltagspraxis handelt es sich dabei um ein zweckmäßig organisiertes Gebilde zumeist aus Holz, an das man sich etwa setzt, um darauf zu arbeiten; in dieser Hinsicht, ist' das dann ein Tisch. Im Kontext der Kernphysik ,ist ‘ derselbe Gegenstand demgegenüber kein Tisch, sondern ein relativ

21 „Der rationalistische Appell, die Sache gemeinsam von allen Seiten durch Experiment und Beobachtung entscheiden zu lassen, ist weder unbefangen noch uneigennützig: er setzt voraus, daß sich der Gegner a limine die Weltanschauung zu eigen machen muß, innerhalb deren [sic] Experiment und Beobachtung einen Sinn haben; somit müßte dieser aber den eigenen Rationalismus und zudem seine ganze weltanschauliche Position preisgeben, noch ehe er dafür hätte kämpfen können“ (Kondylis 1981: 45).

22 „À la différence d'un litige, un différend serait un cas de conflit entre deux parties (au moins) qui ne pourrait pas être tranché équitablement faute d'une règle de jugement applicable aux deux argumentations.“ (Lyotard 1983: 9 bzw. 1987: 9: „Im Unterschied zu einem Rechtsstreit [litige] wäre ein Widerstreit [différend] ein Konfliktfall zwischen (wenigstens) zwei Parteien, der nicht angemessen entschieden werden kann, da eine auf beide Argumentationen anwendbare Urteilsregel fehlt."). 
stabiles Konglomerat von Elementarteilchen, während er für die Polizei vielleicht ein Tatort und für die Kunstgeschichte der Repräsentant eines bestimmten Möbel-Stils ,ist‘ (vgl. Benoist 2011: 60-62).

Die archäologische These von der Zuwanderung über die Beringsee ,ist‘ demzufolge die wissenschaftsintern zwingende Erklärung des Vorhandenseins nordamerikanischer Indianer; der Büffel-Mythos hingegen ,ist“ eine Möglichkeit, deren Identität als eigenständiges Volk stark zu machen. Naturwissenschaftlich ist ersteres von primärem Belang, während letzteres sich als kulturwissenschaftlich ertragreicher erweisen mag. Beide Argumentationen folgen aber nicht ein und derselben Intentionalität und lassen sich daher nicht an einander messen: Die eine Sichtweise ist der anderen nicht als solche überlegen, sondern je abhängig von den eigenen Voraussetzungen und Interessen, und beide erbringen ihre Leistung auf sehr unterschiedlichen Wegen. Diesen Widerstreit gilt es zu begreifen und zu akzeptieren, anstatt der einen oder der anderen Seite alleine Recht zu geben.

\section{Rationalität}

Zumindest aus einem Grund kann von wirklicher Gleichrangigkeit zwischen rationaler Wissenschaftlichkeit und alternativen Formen des Weltverständnisses dennoch keine Rede sein: Allein das wissenschaftlich generierte Wissen besitzt ein - freilich oft zu wenig ausgeschöpftes - Talent zur Selbstreflexivität und ist demgemäß in der Lage, die eigenen Grenzen bzw. Schwachstellen offen zu legen, das heißt einen eventuellen Widerstreit zu bemerken und ernst zu nehmen. Nichts als das rationale Denken kann den Glauben daher als solchen berücksichtigen und in dessen Funktionalität akzeptieren, also sich der Leistungsfähigkeit gerade auch des Nichtrationalen versichern; umgekehrt gelingt kaum mehr als ein mehr oder weniger aktives Ignorieren. Demzufolge ist auch nur das rationale respektive wissenschaftliche Denken in der Lage, alternativ organisierten Glaubensüberzeugungen auf den Grund zu gehen und sie in ihrer immanenten Logik zu problematisieren. Dass archäologische Argumente für das mythische Denken höchstens bestreitbar, nicht aber widerlegbar sind, weiß man in Europa seit dem 18. Jahrhundert, als namentlich die zahllosen Fossilienfunde unabweisbar machten, dass der biblische Schöpfungsbericht zumindest keine buchstäbliche Wahrheit mehr behaupten konnte, weil die Welt offenbar erheblich älter war als die damals errechneten sechs Jahrtausende.

Die vorrangige Erklärung für diese überlegene Leistungsfähigkeit der Wissenschaft ist zweifellos in ihrer Rationalität zu suchen, die als solche zur Grundausstattung unserer Gattung gehört und zwar nicht ohne historisch-kulturelle Modifikationen auskommt, überall aber auf den gleichen Regeln der Logik beruht. In dieser speziellen Hinsicht braucht man zwischen Naturwissenschaften und Geisteswissenschaften nicht zu unterscheiden: In allen Fällen wird methodengeleitet gearbeitet, 
sodass es immer möglich sein muss, Auskunft über das jeweilige Vorgehen zu geben und zu begründen, warum man es so und nicht anders macht.

Was Wissenschaft vordergründig an Wissen produziert, ist demnach zumeist mehr Vermutung als Gewissheit, mehr provisorisch als überzeitlich. Dieses Wissen im strengen Sinn kann seiner Unwahrscheinlichkeit wegen nicht der eigentliche Hauptzweck wissenschaftlichen Arbeitens sein, und genau das ist das Gute an der Sache: Das wirklich Wissenschaftliche an der Wissenschaft liegt weniger in ihren jeweiligen Ergebnissen von meist kurzer Halbwertzeit als vielmehr in den selbstkritischen Strategien, die ihrer Arbeit zugrunde liegen oder doch zumindest zugrunde liegen sollten, damit das Gebot der Rationalität sachgerecht zum Tragen kommt. Fehler bzw. Irrtümer gehören dabei wesentlich zum System und sind daher kein Mangel, sondern der eigentliche Motor. Erkenntnis ist insofern stets von ihrer Umgebung abhängig: Unausweichlich steht sie in Zusammenhängen, aus denen heraus erst sich ihre Gültigkeit, ihre Überzeugungskraft ergibt; diese Zusammenhänge sind selbst wiederum gewissermaßen flüssig, kaum je exakt zu erfassen und allemal unscharf.

\section{Wissen und Wissenschaftlichkeit}

Um mit Recht und Vernunft von ,Wissen“ reden zu dürfen, kommt es also weniger darauf an, ob bestimmte Erkenntnisse tatsächlich ,wahr' sind und auf immer und ewig in Geltung bleiben werden. Entscheidend ist vielmehr, dass es sich um methodisch reflektierte Denkinhalte handelt, über deren Zustandekommen man sich verständigen kann, weil sich Verfahren wie Resultate gleichermaßen der Überprüfung aussetzen; mehr wird sich an ,Objektivität‘ nur selten erzielen lassen. Wissenschaft ist demzufolge nicht anders als prozessual $\mathrm{zu}$ begreifen, das heißt als beständige Selbstkorrektur in der Erwartung, aufgrund neuer Beobachtungen künftig ganz und gar anders denken und untersuchen zu müssen; tiefgreifende Umbrüche wie der Austausch des ptolemäischen gegen das kopernikanische Weltbild oder der Übergang von der mechanistischen Physik des 19. Jahrhunderts zur Relativitätstheorie belegen das drastisch genug. Alles Wissen bleibt insofern sinnvollerweise vorläufig und muss sich immer neu zur Disposition stellen, wenn es für den Augenblick als Wissen gelten will. An die Pathosformel Wahrheit braucht Wissen daher nicht ohne Not gebunden zu werden. Es genügt wohl, in all den Fällen von Wissen zu sprechen, in denen eine bestimmte Aussage hinreichend begründet ist: dass also erklärbar ist, woher man seine Aussage hat und warum man sie zu wissen behauptet. Daran glauben muss sonst freilich niemand, aber man sollte doch einen mindestens ebenso guten Grund nennen können, wenn man sich von Fall zu Fall lieber zurückhält.

Gerade weil das Erkennen, wie Niklas Luhmann (1990: 16) betont hat, nun einmal „nur aufgrund der Möglichkeit des Sich-Irrens zustande“ kommt und durch Wissen- 
schaft daher „nicht Sicherheit, sondern gerade Unsicherheit gesteigert“ wird, ${ }^{23}$ weist die Wissenschaftlichkeit eine Dynamik auf, und eben diese ist es, worauf sich mit guten Gründen und ebenso gutem Gewissen nun wirklich vertrauen lässt (bei aller aufgeklärten Skepsis im Detail). Um es abschließend mit dem amerikanischen Wissenschaftsironiker Richard Rorty (1991: 34) zu sagen: „But there is nothing wrong with science, there is only something wrong with the attempt to divinize it [...]“. Zur Vergötterung von Wissenschaft bzw. Wissenschaftlichkeit besteht in der Tat keinerlei Anlass, da sie zum Glück Menschenwerk ist.

\section{Literatur}

Benoist, J. (2011). Éléments de philosophie réaliste: Réflexions sur ce que l'on a. Paris: Vrin.

Benoist, J. (2014). Elemente einer realistischen Philosophie: Reflexionen über das, was man hat. Aus dem Französischen von David Espinet. Berlin: Suhrkamp.

Benoist, J. (2017). L'adresse du réel. Paris: Vrin.

Boghossian, P. (2006). Fear of Knowledge: Against Relativism and Constructivism. Oxford: Oxford University Press.

Ferraris, M. (2012). Manifesto del nuovo realismo. Roma, Bari: Gius. Laterza \& Figli.

Ferraris, M. (2014). Manifest des Neuen Realismus (Recht als Kultur 6). Aus dem Italienischen von Malte Osterloh. Frankfurt/Main: Vittorio Klostermann.

Feyerabend, P. (2010 [1975]). Against Method: Outline of an Anarchistic Theory of Knowledge. $4^{\text {th }}$ edition. Introduced by lan Hacking. London, New York: Verso.

Husserl, E. (2012 [1954]). Die Krisis der europäischen Wissenschaften und die transzendentale Phänomenologie: Eine Einleitung in die phänomenologische Philosophie. Mit einer Einleitung und Registern hrsg. von Elisabeth Ströker. Hamburg: Felix Meiner.

Johnson, G. (1996). Indian Tribes' Creationists Thwart Archeologists. The New York Times, October 22 ${ }^{\text {nd }}, 1996$ [https://www.nytimes.com/1996/10/22/science/indian-tribes-

creationists-thwart-archeologists.html; 15.03.2019].

Kant, I. (1781). Critik der reinen Vernunft. Riga: Johann Friedrich Hartknoch.

Kant, I. (1784). Beantwortung der Frage: Was ist Aufklärung?. F. Gedike und J. E. Biester. Hrsg. Berlinische Monatsschrift. Vierter Band. Julius bis December 1784. Berlin: Haude und Spener, 481-494.

Kondylis, P. (1981). Die Aufklärung im Rahmen des neuzeitlichen Rationalismus. Stuttgart: Ernst Klett.

Lessing, G. E. (1778). Eine Duplik. Braunschweig: Buchhandlung des Fürstlichen Waisenhauses. Luhmann, N. (1990). Die Wissenschaft der Gesellschaft. Frankfurt/Main: Suhrkamp.

Lyotard, J.-F. (1983). Le Différend. [Paris]: Les Éditions de Minuit.

Lyotard, J.-F. (1987). Der Widerstreit (Supplemente 6). Übersetzt von Joseph Vogl. München: Wilhelm Fink.

23 „Das wissenschaftliche Wissen ist weniger sicher als das Alltagswissen. In der Interpretation von Wahrnehmungen des Alltags entstehen normalerweise keine Zweifel. Eine Rose, die man sieht, ist eine Rose, oder jedenfalls doch eine Blume. Ganz anders die Interpretation der Ergebnisse von Experimenten oder sonstigen wissenschaftlichen ,Daten'. Durch Wissenschaft wird nicht Sicherheit, sondern gerade Unsicherheit gesteigert - in gerade noch tolerierbaren Grenzen“ (Luhmann 1990: 325). 
Meillassoux, Q. (2006). Après la finitude: Essai sur la nécessité de la contigence. Mit einem Vorwort von Alain Badiou. Édition revue. Paris: Seuil.

Meillassoux, Q. (2008). Nach der Endlichkeit: Versuch über die Notwendigkeit der Kontingenz. Aus dem Französischen von Roland Frommel. Zürich, Berlin: diaphanes.

Nietzsche, F. (1980 [1873]). Ueber Wahrheit und Lüge im aussermoralischen Sinne. In: G. Colli und M. Montinari. Hrsg. F. Nietzsche: Sämtliche Werke. Kritische Studienausgabe in 15 Bänden: Band 1. München: Deutscher Taschenbuch Verlag, 873-890.

Putnam, H. (2012). Philosophy in an Age of Science: Physics, Mathematics, and Skepticism. Hrsg. von M. De Caro und D. Macarthur. Cambridge, London: Harvard University Press.

Rorty, R. (1991). Solidarity or Objectivity. In: idem. Objectivity, Relativism, and Truth. Philosophical papers, Bd. 1. Cambridge: Cambridge University Press, 21-34. 
